\title{
Carpal Tunnel Syndrome in a patient with Riche- Cannieu Anastomosis
}

\author{
Mervat Wahba* \\ Department of Neurology, University of Tennessee Health Sciences Center, USA
}

*Corresponding author: Mervat Wahba, Associate Professor of Neurology,

University of Tennessee Health Sciences Center, Tennessee, USA.

Received Date: September 24, 2018

Published Date: October 15, 2018

\section{Introduction}

The intrinsic hand muscles are innervated by the ulnar and median nerves. There are anatomic anomalous innervations in the upper extremities that every electromyographer should be able to identify as these may be commonly encountered. The presence of these anomalies may lead to challenges in interpreting electrophysiological studies and during surgical operations. Furthermore, the symptoms of carpal tunnel syndrome may be diminished or accentuated by the presence of these anomalies.

\section{Riche-Cannieu Anastomosis (RCA)}

The hand muscles are innervated by the median and ulnar nerves. The median nerve innervates the abductor pollicis Brevis, opponens Pollicis, the superficial head of the flexor pollicis brevis, the first and second lumbricals. The ulnar nerve innervates the hypothenar muscles, the interossei, the third and fourth lumbricals, the adductor pollicis, and the deep head of the flexor pollicis brevis.

Riche-Cannieu Anastomosis was independently described a palmar ulnar-to-median nerve crossover in the hand at the thenar eminence by Riche [1] and Cannieu [2]. In RCA, there is a neural connection between the deep branch of the ulnar nerve and the recurrent branch of the median nerve that supplies motor axons to the abductor pollicis brevis or opponens pollicis muscle. The condition is believed to be prevalent in a large percentage of individuals, the reported incidence reflects large discrepancy in the medical literature. Cannieu [2] reported an incidence of $13 \%$. Harness and Sekeles [3] found RCA in 28 of 35 dissected hands (77\%). Electrophysiologiocally, RCA was reported in $1.4 \%$ of electromyography reports dedicated to studying focal neuropathies in the upper extremities [4].

Carpal tunnel syndrome (CTS) is one of the most frequent entrapment neuropathies, described as a symptomatic compression neuropathy of the median nerve at the level of the wrist. The incidence rate is estimated to be $276 / 100,000$ [5], with a prevalence rate of $9.2 \%$ in women, $6 \%$ in men [6]. An estimated $20 \%$ of individuals complaining of numbness and tingling in the hands are expected to have carpal tunnel syndrome [7]. Numerous variations and refinements of different techniques for electrophysiological testing for CTS have occurred over the years. There has been emphasis on comparing the median sensory response to that of the ulnar and radial responses, and to comparing the more distal portion of the median sensory response to that across the carpal ligament. The "standard techniques" include absolute distal sensory latency between wrist and digit, and the absolute distal motor latency between wrist and thenar muscles. A combination of the following approaches exist for assessing median nerve entrapment:

1. Median-ulnar mixed nerve latency difference to the midpalm.

2. Median-radial sensory latency difference to the thumb.

3. Median-ulnar sensory latency difference to the ring finger.

4. Median mid-palmar sensory latency compared to wrist latency

5. Short segment sensory stimulation across the carpal tunnel (inching).

6. Robinson cumulative summary index (CSI)

7. Median (to lumbrical) - ulnar (to interosseous) motor latency difference (LILD) across the carpal tunnel

8. Median-thenar to ulnar-thenar motor latency difference (TTLD)

(Williams and Andary AANEM 2001) [8]. 
In patients with RCA, on nerve conduction studies, a low amplitude median motor response is observed, the abnormality is out of proportion to the median sensory and median mixed nerve potential abnormalities which may be normal, or abnormal if there is carpal tunnel syndrome. In some cases, the median nerve action potential is absent on recording from the thenar muscles as they are innervated by the ulnar nerve, the thenar muscle electromyography is unaffected.

In individuals with RCA who develop ulnar neuropathy proximal to the anomalous crossover, abnormal needle exam may be observed both the median and ulnar-innervated muscles, which may lead to a misdiagnosis of medial cord, lower plexus, $\mathrm{C}_{8}$ or $\mathrm{T}_{1}$ roots, or concomitant median and ulnar neuropathies [9].

If a median neuropathy is situated proximal to the ulnarto-median anastomosis in a patient with an unidentified RCA, it will be underestimated. In patients with RCA and concomitant significant median neuropathy, it may be interpreted as partial median neuropathy as some of the median-innervated muscles as innervated by the ulnar nerve through the crossover branch.

\section{Other upper extremity anomalous innervation}

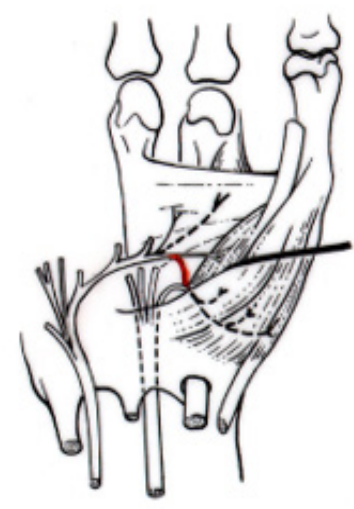

Figure 1: Riche-Cannieu anastomosis.

A commonly encountered anatomic anomaly is MartinGruber Anastomosis (MGA), a median-to-ulnar anastomosis. Electrophysiological studies reported an incidence of 15-32\% [10]. Another anomaly is Berretini Anastomosis, a communication between the ulnar fourth common digital nerve and the median third common digital nerve. The incidence of this anastomosis is over $80 \%$ [11] (Figure 1).

\section{Case Report}

A 59-year-old woman was referred to the EMG lab department for evaluation of suspected carpal tunnel syndrome manifesting as bilateral paresthesias in the lateral three digits on the palmar aspect. Nerve conduction studies showed delay left and right sensory nerve action potentials (SNAP) and delay in the mixed nerve action potentials (NAP) bilaterally, where the median NAP was recorded at $2.5 \mathrm{msec}, 2.6 \mathrm{msec}$ on the right and left median nerves respectively, where the upper normal is $2.2 \mathrm{msec}$.
On the right side, the median distal motor latency (DML) was normal. On the left side, the median compound motor action potential (CMAP) was nonrecordable with no clinical atrophy of the left thenar muscle. The left lumbrical II-second interosseous comparison showed a delay on stimulating the left median nerve. Needle electromyography was normal. These findings were consistent with bilateral carpal tunnel syndrome, plus a left RicheCannieu anastomosis (Figure 2).

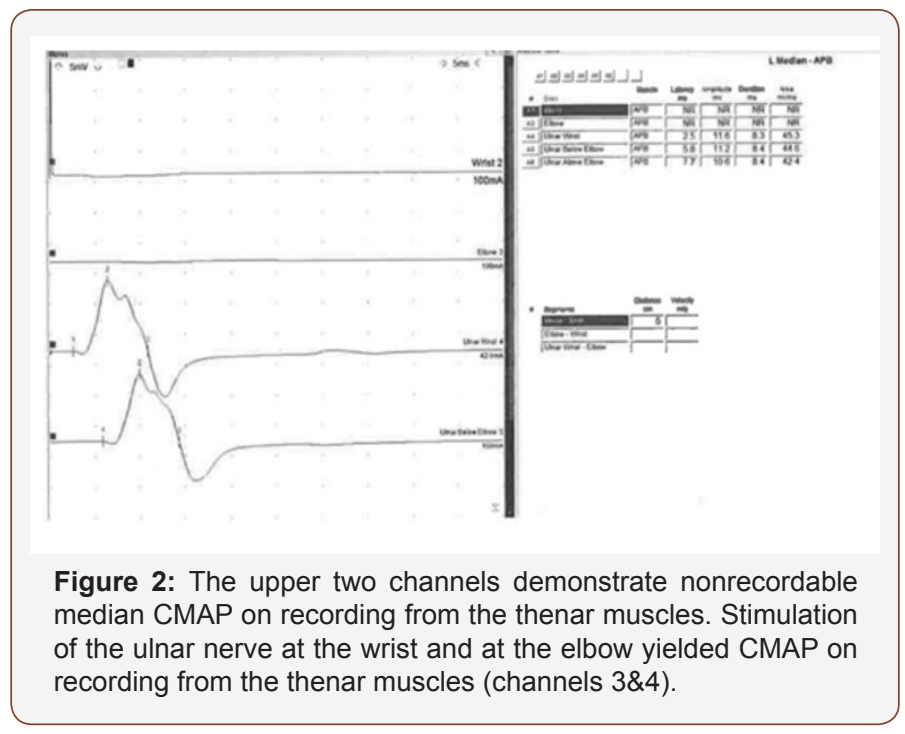

The left median nerve CMAP was non-recordable on recording from the thenar muscles. This left median CMAP finding associated with a recordable left median SNAP and mixed nerve action potential supported the diagnosis of Riche-Cannieu anastomosis. Paradoxically low or absent median CMAP in isolation should raise the question of Riche-Cannie anastomosis, as this may be misinterpreted as very severe carpal tunnel syndrome.

\section{Discussion}

In this case, a recordable median sensory potential and a mixed nerve action potential were obtained on the left. The left median compound motor action potential was non-recordable. The needle EMG was normal bilaterally. Technical errors were excluded. These findings are consistent with RCA anomalous innervation in the left palm. Low median motor amplitudes in isolation may be erroneously diagnosed as severe CTS if anomalous innervations are not sought after Also, the above findings may be interpreted as an intra-spinal canal lesion or anterior horn cell disorder in the absence of EMG findings.

\section{Conclusion}

Nerve anastomoses between the median and ulnar nerves may cause attenuation or exacerbation of the clinical symptoms and should be investigated electrophysiologically. RCA should be suspected when the hand muscles are clinically intact in the presence of a severe median nerve lesion in the forearm. Also, in traumatic cases, a complete lesion of the median nerve at the forearm might erroneously be interpreted as a partial lesion if RCA is not recognized. In every surgical dissection to the hand, this 
anomaly should be kept in mind.

\section{Acknowledgement}

None.

\section{Conflict of interest}

No conflict of interest.

\section{References}

1. Cannieu A (1896) Recherche sur l' innervation de l'eminence thenar par le cubital. JMed Bordeaux: 377-379.

2. Riché D (1897) Le nerf cubital et les muscles de l'eminence thenar. Bull Mem SocAnat Paris: 251-252.

3. Harness D, Sekeles E (1971) The double anastomotic innervation of thenar muscles. J Anat 109(Pt3): 461-466.

4. Caetano EB, Vieira LA, Nakmichi YC, Sawada MM, de Andrade RA et al. (2017) Cannieu-Riché Anastomosis: New Classification. SM J Clin Anat 1(1): 1001.
5. Mondelli M, Giannini F, Giacchi M (2002) Carpal tunnel incidence in a general population. Neurology 58(2): 289-294.

6. Atroshi I, Gummesson C, Johnsson R, Ornstein E, Ranstam J, et al. (1999) Prevalence of carpal tunnel syndrome in population. JAMA 282(2): 153158.

7. Amirlak B, Upadhyaya K, Ahmed O, Wolff T, Tsai T (2010) Median NervE Entrapment 1-11-2010 Internet Communication.

8. Williams, Andary AANEM, Michael T A (2001) Carpal Tunnel Syndrome: Techniques for Diagnosis Faren H. Williams, MD Moss Rehabilitation Associates.

9. Saperstein DS, King RB (2000) Motor neuron presentation of an ulnar neuropathy and Riche-Cannieu anastomosis. Electromyogr Clin Neurophysiol 40(2): 119-122.

10. Mannerfelt L (1966) Studies on the hand in the ulnar nerve paralysis. A clinical experimental investigation in normal and anomalous innervations. Acta Orthop Scand 87: 23-142

11. Unver Dogan N, Uysal II, Seker M (2009) The communication between the ulnar and median nerves in upper limb. Neuroanatomy 8: 15-19. 